andauern. Es gelangen hauptsächlich Wellen von ca. $3^{\mathrm{m}}$ Dauer und 0.05 Amplitude zur Beobachtung, sie sind demnach in Wahrheit äusserst flach und würden bei einer Länge von $5-600 \mathrm{~km}$ eine Höhe von nur $18 \mathrm{~mm}$ besitzen. In einem besonders merkwürdigen Fall, am 19. Oct. I892, welcher in meiner Anfangs citirten Abhandlung dargestellt ist, wurden aufeinanderfolgend ungefähr 200 dieser Wellen

Merseburg 1893 März. in einem Zeitralım von $10^{h}$ gezählt. Nach den Beobachtungen des Erdbebenforschers Milne in Japan ist anzunehmen, dass das Auftreten dieser und ähnlicher Wellen an steile barometrische Gradienten gebunden ist, wenngleich Näheres über die Entstehung derselben zur Zeit nicht bekannt ist.

\title{
Elementi ed effemeride di (303) Josephina (III. opposizione).
}

Calcolate le perturbazioni per Giove e Saturno e ricorretti gli elementi in modo da ben soddisfare alle due prime opposizioni ottenni il seguente sistema di elementi osculanti per la terza opposizione.

Epoca ed oscul. 1893 luglio 19.0 t. m. Berlino.

$$
\begin{aligned}
& M=23^{\circ} 34^{\prime} 42^{\prime \prime} 8 \\
& \infty=733129.6 \\
& \delta=345 \text { I5 } 6.4 \quad \text { Equ. med. I } 892.0 \\
& i=\begin{array}{lll}
6 & 54 & 24.8
\end{array} \\
& \varphi=33927.6 \\
& \mu=643 " 32802 \\
& \log a=0.4946172 \\
& m_{0}=13.3 \quad g=8.0
\end{aligned}
$$

\begin{tabular}{|c|c|c|c|}
\hline 1893 & $\alpha \mathrm{I} 893.0$ & $\delta$ I 893.0 & $\log \Delta$ \\
\hline Lugl. 4 & $19^{\mathrm{h}} 28^{\mathrm{m}} 5^{8} \cdot 90$ & $-30^{\circ} 28^{\prime} 58^{\prime \prime} 9$ & 0.350549 \\
\hline 88 & $25 \quad 24.26$ & $\begin{array}{lll}30 & 33 & 12.9\end{array}$ & 349453 \\
\hline 612 & 2146.99 & $3^{\circ} 3^{6} \quad 7.8$ & 349230 \\
\hline 16 & $18 \quad 10.70$ & $\begin{array}{lll}30 & 37 & 36.4\end{array}$ & 349887 \\
\hline 20 & 1439.16 & $\begin{array}{lll}3 \circ & 37 & 39.2\end{array}$ & 351402 \\
\hline 24 & 19 II 16.15 & $\begin{array}{lll}-30 & 3^{6} & 22.2\end{array}$ & 0.353720 \\
\hline
\end{tabular}

Effemeride. $\mathbf{I}^{\text {h }}$ t. m. Berlino.

Coi miei elementi corretti colle osservazioni della seconda opposizione prima del calcolo delle perturbazioni ed inseriti nel B. A. J. del 1895 il luogo per luglio 10.5 è $19^{\mathrm{h}} 23^{\mathrm{m}} 45^{\mathrm{s}}-30^{\circ} 34^{\prime} 5^{\circ}$ e non $19^{\mathrm{h}} 19^{\mathrm{m}} 7$ e $-30^{\circ} 42^{\prime}$ come è calcolato nel B. A. J. E sperabile ed anche probabile che il luogo dato dalla precedente effemeride sia assai prossimo al luogo vero.

Roma 1893 giugno 3 .

E. Millosevich.

\section{Aufforderung betr. Beobachtungen des Winnecke'schen Cometen $1892 \mathrm{IV}$.}

Prof. E. Frh. v. Haerdtl in Innsbruck bittet um baldige Veröffentlichung von noch rückständigen Beobachtungen der letzten Erscheinung (1892 IV) des Winnecke'schen Cometen.

Kr.

\section{Versammlung der Astronomischen Gesellschaft.}

Nach Beschluss der Münchener Versammlung sollte in diesem Jahre die Astronomische Gesellschaft in Utrecht tagen. Der Vorstand glaubt indessen in Anbetracht der sanitären Verhältnisse in Mitteleuropa und insbesondere in Rücksicht auf die hierdurch voraussichtlich entstehenden Verkehrshindernisse den allgemeinen Wünschen entgegen zu kommen, wenn er von der Abhaltung der Astronomenversammlung in diesem Jahre absieht. Dieselbe wird also erst im Jahre 1894 stattfinden.

1893 Juni.

H. Gyldén, Vorsitzender,

R. Lehmann-Filhés, H. Seeliger, Schriftfuhrer.

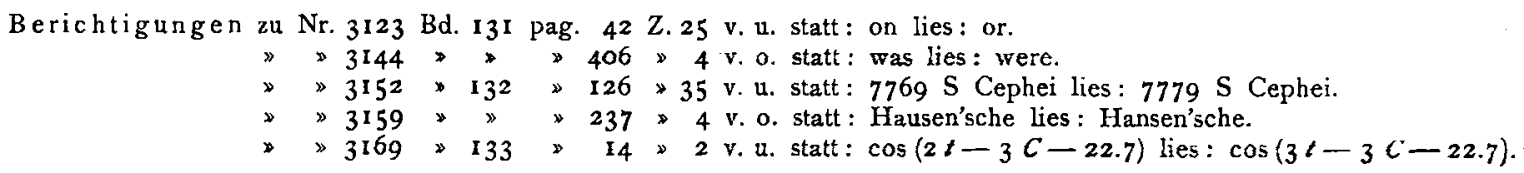

Inhalt zu Nr. 3169. E. von Rebeur-Pasthwits. Ueber die Möglichkeit, die Existenz von Mondgliedern in der scheinbaren täglichen Oscillation der Lothlinie nachzuweisen. 1. - E. Millosevich. Elementi ed effemeride di (303) Josephina (II. opposizione). 23. - Aufforderung betr. Beobachtungen des Winnecke'schen Cometen 1892 IV. 23. - Versammlung der Astronomischen Gesellschaft. 23. - Berichtigungen. 23. 